\title{
Two-component generalizations of the Novikov equation
}

Hongmin Li

To cite this article: Hongmin Li (2019) Two-component generalizations of the Novikov equation, Journal of Nonlinear Mathematical Physics 26:3, 390-403, DOI: https://doi.org/10.1080/14029251.2019.1613048

To link to this article: https://doi.org/10.1080/14029251.2019.1613048

Published online: 04 January 2021 


\title{
Two-component generalizations of the Novikov equation
}

\author{
Hongmin Li \\ Schools of Mathematics, Huaqiao University, \\ Quanzhou, Fujian 362021, P R China \\ lihongmin@hqu.edu.cn
}

Received 1 November 2018

Accepted 2 March 2019

\begin{abstract}
Some two-component generalizations of the Novikov equation, except the Geng-Xue equation, are presented, as well as their Lax pairs and bi-Hamiltonian structures. Furthermore, we study the Hamiltonians of the GengXue equation and discuss the homogeneous and local properties of them.
\end{abstract}

Keywords: Camassa-Holm type equation; Lax pair; Bi-Hamiltonian structure

2000 Mathematics Subject Classification: 37K10, 35Q51, 35Q58

\section{Introduction}

In recent decades, the Camassa-Holm $(\mathrm{CH})$ type equations raised a lot of interest because of their specific properties, one of which is that they possess peakon solutions (peaked soliton solutions with discontinuous derivatives at the peaks). The most celebrated member of them is the $\mathrm{CH}$ equation [1]

$$
m_{t}+u m_{x}+2 u_{x} m=0, \quad m=u-u_{x x},
$$

which was found to be completely integrable with a Lax pair and associated bi-Hamiltonian structure $[1,2]$, and related by a reciprocal transformation to the first negative flow in the KdV hierarchy [18]. It is worthwhile to note that an inverse scattering approach for the $\mathrm{CH}$ equation was developed in the Refs. $[5,11,14]$, which showed that after a suitable change of variables, the $\mathrm{CH}$ flow becomes a linear flow at constant speed, this being the strong version of integrability (the infinite-dimensional counterpart of the classical Liouville theorem). Moreover, while an initial $\mathrm{m}$ without change of sign leads to solutions of $\mathrm{CH}$ that are defined for all times $t \geq 0[3,8]$, among the initial $m$ that change sign one encounters solutions of $\mathrm{CH}$ that model breaking waves, in the sense that the solution itself remains bounded but its slope becomes unbounded [10].

In 1999, Degasperis and Procesi used an asymptotic integrability approach to isolate integrable third-order equations, and discovered a new $\mathrm{CH}$ type equation, i.e., the Degasperis-Procesi (DP) equation

$$
m_{t}+u m_{x}+3 u_{x} m=0, \quad m=u-u_{x x},
$$

which turns out to be integrable with a bi-Hamiltonian structure and a Lax pair [16,17], and is reciprocal linked to a negative flow in the Kaup-Kupershmidt hierarchy [22]. Furthermore, the inverse scattering problem for the DP equation was formulated in Ref. [12]. Especially, for CH and DP equations one can prove that the peakons are orbitally stable [15, 25,31], in the sense that their shape is stable under small perturbations, and therefore these patterns are detectable. This is an 
essential aspect since both equations arise as models for water waves [13, 23, 24]. Note that the travelling wave solutions of greatest height of the governing equations for water waves have a peak at their crest $[6,7,9,35]$. The proof of the orbital stability of the peakons relies on the conservation laws for $\mathrm{CH}$ and DP.

Both the $\mathrm{CH}$ and DP equations are the third order $\mathrm{CH}$ type equations with quadratic nonlinearity. Recently, by the symmetry classification study of nonlocal partial differential equations with quadratic or cubic nonlinearity, Novikov discovered a new $\mathrm{CH}$ type equation with cubic nonlinearity [32]

$$
m_{t}+u^{2} m_{x}+3 u u_{x} m=0, \quad m=u-u_{x x} .
$$

Subsequently, Hone and Wang presented a Lax pair for the Novikov equation (1.3) and proved it is related to the negative flow in the Sawada-Kotera hierarchy by a reciprocal transformation. Infinitely many conserved quantities and a bi-Hamiltonian structure of the Novikov equation are also constructed [21].

Later Geng and Xue [19] presented a two-component generalization for the Novikov equation

$$
\begin{aligned}
& m_{t}+3 u_{x} v m+u v m_{x}=0, \\
& n_{t}+3 v_{x} u n+u v n_{x}=0, \\
& m=u-u_{x x}, \quad n=v-v_{x x},
\end{aligned}
$$

which admits a Lax representation

$$
\varphi_{x}=\bar{M} \varphi, \quad \varphi_{t}=\bar{N} \varphi
$$

where

$$
\begin{aligned}
& \bar{M}=\left(\begin{array}{ccc}
0 & m \lambda & 1 \\
0 & 0 & n \lambda \\
1 & 0 & 0
\end{array}\right), \\
& \bar{N}=\left(\begin{array}{ccc}
\frac{1}{3 \lambda^{2}}-u_{x} v & \frac{u_{x}}{\lambda}-u v m \lambda & u_{x} v_{x} \\
\frac{v}{\lambda} & -\frac{2}{3 \lambda^{2}}+u_{x} v-u v_{x} & -u v n \lambda-\frac{v_{x}}{\lambda} \\
-u v & \frac{u}{\lambda} & \frac{1}{3 \lambda^{2}}+u v_{x}
\end{array}\right) .
\end{aligned}
$$

They also gave an infinite sequence of conserved quantities of the system (1.4) (also called GengXue equation). Li et al proved it is bi-Hamiltonian [29] and reciprocal linked to a negative flow in the modified Boussinesq hierarchy [30]. Very recently, we constructed a Liouville transformation to connect it with another negative modified Boussinesq equation, and Lax pairs as well as biHamiltonian structures of them are connected [27].

Recently, we make the vector prolongation of the Lax pair (1.5) as follows [28]

$$
\Phi_{x}=\widetilde{M} \Phi, \quad \Phi_{t}=\widetilde{N} \Phi
$$


with

$$
\begin{aligned}
& \tilde{M}=\left(\begin{array}{ccc}
0 & \lambda Q^{T} & 1 \\
\mathbf{0}^{T} & \mathbf{0}_{N} & \lambda R \\
1 & \mathbf{0} & 0
\end{array}\right), \\
& \widetilde{N}=\left(\begin{array}{ccc}
-U_{x}^{T} V & \frac{U_{x}^{T}}{\lambda}-\lambda U^{T} V Q & U_{x}^{T} V_{x} \\
\frac{V}{\lambda} & -\frac{I_{N}}{\lambda^{2}}+V U_{x}^{T}-V_{x} U^{T} & -\frac{V_{x}}{\lambda}-\lambda U^{T} V R \\
-U^{T} V & \frac{U^{T}}{\lambda} & U^{T} V_{x}
\end{array}\right),
\end{aligned}
$$

where $\mathbf{0}$ and $\mathbf{0}_{N}$ are respectively $N$ dimension row vector and $N \times N$ zero matrix and $T$ is the transpose of a vector. $I_{N}$ denotes the $N \times N$ identity matrix and $Q, R, U, V$ are the $N$-component column vector potentials, and $U_{x}^{T}=\frac{\partial U^{T}}{\partial x}$.

Then the zero-curvature equation for (1.7) yields the multi-component Novikov equation

$$
\begin{aligned}
Q_{t} & =-2 U_{x}^{T} V Q-U^{T} V_{x} Q-U^{T} V Q_{x}-Q^{T} V U_{x}+Q^{T} V_{x} U \\
R_{t} & =-2 U^{T} V_{x} R-U_{x}^{T} V R-U^{T} V R_{x}-R^{T} U V_{x}+R^{T} U_{x} V \\
Q & =U-U_{x x}, \quad R=V-V_{x x}
\end{aligned}
$$

which is bi-Hamiltonian [28] proved by the multi-vector method [34]. This multi-component equation can be reduced to Geng-Xue equation (1.4), DP equation (1.2) and Novikov equation (1.3) under the constraints $N=1, Q=m, R=n, U=u, V=v ; N=1, Q=m, U=u, R=V=1$ and $N=1, Q=R=m, V=U=u$ respectively. The aim of this paper is to consider some reductions of the Lax pair (1.7) and the corresponding evolution equations.

This paper is organized as follows. In Section 2, we consider two interesting reductions of the multi-component Novikov equation (1.9) and construct the bi-Hamiltonian structures for them. In Section 3, we compute two infinite sequences of conserved quantities for the Geng-Xue equation, and discuss the homogeneous and local properties of the Hamiltonians of the Geng-Xue equation.

\section{Two-component generalizations of the Novikov equation}

In this section, we will study two reductions of the multi-component Novikov equation (1.9) and obtain two reduced systems, as well as their bi-Hamiltonian structures using Dirac reduction [33].

\subsection{The first two-component generalization of the Novikov equation}

Setting $U=V, Q=R$, the multi-component Novikov equation (1.9) is reduced to

$$
Q_{t}=-3 U_{x}^{T} U Q-U^{T} U Q_{x}-Q^{T} U U_{x}+Q^{T} U_{x} U, \quad Q=U-U_{x x},
$$

which can be transformed to a two-component Novikov equation

$$
\begin{aligned}
q_{t} & =-\left(u^{2}+v^{2}\right) q_{x}-3\left(u u_{x}+v v_{x}\right) q+r\left(u v_{x}-u_{x} v\right), \\
r_{t} & =-\left(u^{2}+v^{2}\right) r_{x}-3\left(u u_{x}+v v_{x}\right) r+q\left(u_{x} v-u v_{x}\right), \\
q & =u-u_{x x}, \quad r=v-v_{x x} .
\end{aligned}
$$

as $Q=R=(q, r)^{T}, U=V=(u, v)^{T}$. Especially when $u=v$, the equation (2.2) is reduced to the Novikov equation (1.3). 
As pointed in [28], the equation (1.9) can be written in the bi-Hamiltonian form

$$
\left(\begin{array}{l}
Q \\
R
\end{array}\right)_{t}=\mathscr{K}\left(\begin{array}{c}
\frac{\delta H_{2}}{\delta Q} \\
\frac{\delta H_{2}}{\delta R}
\end{array}\right)=\mathscr{J}\left(\begin{array}{c}
\frac{\delta H_{1}}{\delta Q} \\
\frac{\delta H_{1}}{\delta R}
\end{array}\right)
$$

using the two compatible Hamiltonian operators

$$
\begin{gathered}
\mathscr{K}=\left(\begin{array}{cc}
\mathbf{0}_{N} & \left(\partial^{2}-1\right) I_{N} \\
\left(1-\partial^{2}\right) I_{N} & \mathbf{0}_{N}
\end{array}\right), \\
\mathscr{J}=\mathscr{J}_{1}+\mathscr{J}_{2}
\end{gathered}
$$

with

$$
\begin{aligned}
& \mathscr{J}_{1}=\left(\begin{array}{c}
\frac{3}{2} Q \partial+Q_{x} \\
\frac{3}{2} R \partial+R_{x}
\end{array}\right)\left(\partial^{3}-4 \partial\right)^{-1}\left(3 Q^{T} \partial+Q_{x}^{T} 3 R^{T} \partial+R_{x}^{T}\right), \\
& \mathscr{J}_{2}=\left(\begin{array}{c}
\frac{1}{2} Q \partial^{-1} Q^{T}+\left(Q \partial^{-1} Q^{T}\right)^{T}-\frac{1}{2} Q \partial^{-1} R^{T}-Q^{T} \partial^{-1} R I_{N} \\
-\frac{1}{2} R \partial^{-1} Q^{T}-R^{T} \partial^{-1} Q I_{N} \frac{1}{2} R \partial^{-1} R^{T}+\left(R \partial^{-1} R^{T}\right)^{T}
\end{array}\right),
\end{aligned}
$$

and the two Hamiltonian functionals

$$
\begin{aligned}
& \mathscr{H}_{1}=\frac{1}{2} \int Q^{T} V+R^{T} U d x \\
& \mathscr{H}_{2}=\frac{1}{2} \int Q^{T} V U_{x}^{T} V-R^{T} U V_{x}^{T} U+\left(R^{T} U_{x}-Q^{T} V_{x}\right) U^{T} V d x .
\end{aligned}
$$

In what follows we will construct the bi-Hamiltonian structure for the multi-component Novikov equation (2.1) from (2.3). Introducing $P=\frac{Q+R}{2}, S=\frac{Q-R}{2}$, the new system with the variables $P, S$ has a Hamiltonian operator:

$$
\Pi(P, S)=\frac{1}{4}\left(\begin{array}{cc}
I_{N} & I_{N} \\
I_{N} & -I_{N}
\end{array}\right) \mathscr{J}\left(\begin{array}{cc}
I_{N} & I_{N} \\
I_{N} & -I_{N}
\end{array}\right)
$$

then after using the Dirac reduction [33] under the constraint $Q=R$ or $S=0$, we have

$$
\Pi(P, 0)=\frac{1}{2}\left(\begin{array}{ll}
\Pi_{11}(P, 0) & \Pi_{12}(P, 0) \\
\Pi_{21}(P, 0) & \Pi_{22}(P, 0)
\end{array}\right),
$$

where $\Pi_{11}(P, 0)=\left(3 Q \partial+2 Q_{x}\right)\left(\partial^{3}-4 \partial\right)^{-1}\left(3 Q^{T} \partial+Q_{x}^{T}\right)+\left(Q \partial^{-1} Q^{T}\right)^{T}-Q^{T} \partial^{-1} Q I_{N}, \Pi_{12}(P, 0)=$ $\Pi_{21}(P, 0)=0, \Pi_{22}(P, 0)=Q \partial^{-1} Q^{T}+\left(Q \partial^{-1} Q^{T}\right)^{T}+Q^{T} \partial^{-1} Q I_{N}$.

So one may obtain the reduced Hamiltonian operator for the multi-component equation (2.1) as 
follows:

$$
\Pi^{\mathrm{red}}(Q)=\frac{1}{2}\left(\Pi_{11}(P, 0)-\Pi_{12}(P, 0)\left[\Pi_{22}(P, 0)\right]^{-1} \Pi_{21}(P, 0)\right)=\frac{1}{2} \Pi_{11}(P, 0) .
$$

To obtain the second Hamiltonian operator for the multi-component equation (2.1), we consider the Hamiltonian operator $\mathscr{K} \mathscr{J}^{-1} \mathscr{K}$ and its Dirac reduction, then the following equality holds:

$$
\Lambda(P, S)=\frac{1}{4}\left(\begin{array}{cc}
I_{N} & I_{N} \\
I_{N} & -I_{N}
\end{array}\right) \mathscr{K} \mathscr{J}^{-1} \mathscr{K}\left(\begin{array}{cc}
I_{N} & I_{N} \\
I_{N} & -I_{N}
\end{array}\right)
$$

Similarly, after the Dirac reduction, one may get

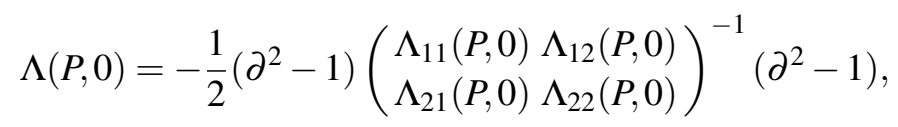

with $\Lambda_{11}(P, 0)=\Pi_{22}(P, 0), \Lambda_{12}(P, 0)=\Lambda_{21}(P, 0)=0$ and $\Lambda_{22}(P, 0)=\Pi_{11}(P, 0)$. So we have

$$
\Lambda(P, 0)=-\frac{1}{2}\left(\partial^{2}-1\right)\left(\begin{array}{cc}
{\left[\Pi_{22}(P, 0)\right]^{-1}} & 0 \\
0 & {\left[\Pi_{11}(P, 0)\right]^{-1}}
\end{array}\right)\left(\partial^{2}-1\right)
$$

which means the second Hamiltonian operator for the equation (2.1) is

$$
\Lambda^{\mathrm{red}}(Q)=-\frac{1}{2}\left(\partial^{2}-1\right)\left[\Pi_{22}(P, 0)\right]^{-1}\left(\partial^{2}-1\right)
$$

Specially, from the constraint $Q=R=(q, r)^{T}, U=V=(u, v)^{T}$ and the Hamiltonian pair (2.7-2.8), the two-component system (2.2) possesses the bi-Hamiltonian operators

$$
\begin{aligned}
& \Pi^{\mathrm{red}}(q, r)=\left(\begin{array}{c}
3 q \partial+2 q_{x} \\
3 r \partial+2 r_{x}
\end{array}\right)\left(\partial^{3}-4 \partial\right)^{-1}\left(3 q \partial+q_{x}, 3 r \partial+r_{x}\right) \\
& +\left(\begin{array}{c}
r \\
-q
\end{array}\right) \partial^{-1}(-r, q) \\
& \Lambda^{\mathrm{red}}(q, r)=-\frac{1}{2}\left(\partial^{2}-1\right)\left(\begin{array}{cc}
3 q \partial^{-1} q+r \partial^{-1} r & q \partial^{-1} r+r \partial^{-1} q \\
q \partial^{-1} r+r \partial^{-1} q & q \partial^{-1} q+3 r \partial^{-1} r
\end{array}\right)^{-1}\left(\partial^{2}-1\right) .
\end{aligned}
$$

Remark 2.1. The two-component system (2.2) appears in the bi-Hamiltonian form

$$
\left(\begin{array}{l}
q \\
r
\end{array}\right)_{t}=\Pi^{\mathrm{red}}(q, r)\left(\begin{array}{c}
\frac{\delta H}{\delta q} \\
\frac{\delta H}{\delta r}
\end{array}\right)=\Lambda^{\mathrm{red}}(q, r)\left(\begin{array}{c}
\frac{\delta W}{\delta q} \\
\frac{\delta W}{\delta r}
\end{array}\right),
$$

with Hamiltonian pair $\Pi^{\mathrm{red}}(q, r), \Lambda^{\mathrm{red}}(q, r)$ given by (2.9). The associated Hamiltonian functional is $H=\frac{1}{2} \int q u+r v d x$, and $W$ is nonlocal and looks very complicated, so we omit it. 


\subsection{The second two-component generalization of the Novikov equation}

Assuming $N$ is an even number, we consider the constraint

$$
Q=\left(\begin{array}{l}
Q_{1} \\
Q_{2}
\end{array}\right), \quad R=\left(\begin{array}{l}
R_{1} \\
R_{2}
\end{array}\right), U=\left(\begin{array}{c}
U_{1} \\
U_{2}
\end{array}\right), \quad V=\left(\begin{array}{c}
V_{1} \\
V_{2}
\end{array}\right)
$$

where $R_{1}=Q_{2}, R_{2}=Q_{1}, V_{1}=U_{2}, V_{2}=U_{1}$ and all of them are $\frac{N}{2}$ dimension column vectors, then the multi-component Novikov equation (1.9) reduces to

$$
\begin{aligned}
& Q_{1 t}=- 3\left(U_{1}^{T} U_{2}\right)_{x} Q_{1}-2 U_{1}^{T} U_{2} Q_{1 x}+Q_{1}^{T}\left(U_{2 x} U_{1}-U_{2} U_{1 x}\right) \\
&+Q_{2}^{T}\left(U_{1 x} U_{1}-U_{1} U_{1 x}\right), \\
& Q_{2 t}=- 3\left(U_{1}^{T} U_{2}\right)_{x} Q_{2}-2 U_{1}^{T} U_{2} Q_{2 x}+Q_{1}^{T}\left(U_{2 x} U_{2}-U_{2} U_{2 x}\right) \\
&+Q_{2}^{T}\left(U_{1 x} U_{2}-U_{1} U_{2 x}\right) \\
& Q_{1}=U_{1}-U_{1 x x}, Q_{2}=U_{2}-U_{2 x x} .
\end{aligned}
$$

In particular, setting $Q=(q, r)^{T}, R=(r, q)^{T}, U=(u, v)^{T}, V=(v, u)^{T}$, the multi-component equation (2.12) leads to another two-component Novikov equation

$$
\begin{aligned}
& q_{t}=-2 u v q_{x}-2 u v_{x} q-4 u_{x} v q, \\
& r_{t}=-2 u v r_{x}-2 u_{x} v r-4 u v_{x} r, \\
& q=u-u_{x x}, \quad r=v-v_{x x},
\end{aligned}
$$

which may be reduced to the Novikov equation (1.3) under the constraint $u=v$.

To construct the bi-Hamiltonian structure of the multi-component system (2.12), we apply the same procedure in subsection 2.1 and introduce these symbols $P_{1}=\frac{Q_{1}+R_{2}}{2}, P_{2}=\frac{Q_{2}+R_{1}}{2}, S_{1}=\frac{Q_{1}-R_{2}}{2}$, $S_{2}=\frac{Q_{2}-R_{1}}{2}$, then the Hamiltonian operator $\Pi\left(P_{1}, P_{2}, S_{1}, S_{2}\right)$ for the new system with the variables $P_{1}, P_{2}, S_{1}, S_{2}$ can be written as:

$$
\Pi\left(P_{1}, P_{2}, S_{1}, S_{2}\right)=\frac{1}{4} \mathscr{F} \mathscr{J} \mathscr{F}^{*}
$$

where $\mathscr{F}=\left(\begin{array}{cccc}I & 0 & 0 & I \\ 0 & I & I & 0 \\ I & 0 & 0 & -I \\ 0 & I & -I & 0\end{array}\right), 0$ and $I$ are respectively $\frac{N}{2} \times \frac{N}{2}$ zero matrix and $\frac{N}{2} \times \frac{N}{2}$ identity matrix.

After using the Dirac reduction under the constraint $Q_{1}=R_{2}, Q_{2}=R_{1}$ or $S_{1}=S_{2}=0$, the operator $\Pi\left(P_{1}, P_{2}, S_{1}, S_{2}\right)$ reduces to

$$
\begin{aligned}
& \Pi\left(P_{1}, P_{2}, 0,0\right)= \\
& \left(\begin{array}{cccc}
\Pi_{11}\left(P_{1}, P_{2}, 0,0\right) & \Pi_{12}\left(P_{1}, P_{2}, 0,0\right) & 0 & 0 \\
\Pi_{21}\left(P_{1}, P_{2}, 0,0\right) & \Pi_{22}\left(P_{1}, P_{2}, 0,0\right) & 0 & 0 \\
0 & 0 & \Pi_{33}\left(P_{1}, P_{2}, 0,0\right) & \Pi_{34}\left(P_{1}, P_{2}, 0,0\right) \\
0 & 0 & \Pi_{43}\left(P_{1}, P_{2}, 0,0\right) & \Pi_{44}\left(P_{1}, P_{2}, 0,0\right)
\end{array}\right)
\end{aligned}
$$


with

$$
\begin{aligned}
\Pi_{11}\left(P_{1}, P_{2}, 0,0\right)= & \frac{1}{2}\left(3 Q_{1} \partial+2 Q_{1 x}\right)\left(\partial^{3}-4 \partial\right)^{-1}\left(3 Q_{1}^{T} \partial+Q_{1 x}^{T}\right)+\frac{1}{2}\left(Q_{1} \partial^{-1} Q_{1}^{T}\right)^{T}, \\
\Pi_{12}\left(P_{1}, P_{2}, 0,0\right)= & \frac{1}{2}\left(3 Q_{1} \partial+2 Q_{1 x}\right)\left(\partial^{3}-4 \partial\right)^{-1}\left(3 Q_{2}^{T} \partial+Q_{2 x}^{T}\right)+\frac{1}{2}\left(Q_{2} \partial^{-1} Q_{1}^{T}\right)^{T} \\
& \quad-\frac{1}{2}\left(Q_{1}^{T} \partial^{-1} Q_{2}+Q_{2}^{T} \partial^{-1} Q_{1}\right) I, \\
\Pi_{21}\left(P_{1}, P_{2}, 0,0\right)= & \frac{1}{2}\left(3 Q_{2} \partial+2 Q_{2 x}\right)\left(\partial^{3}-4 \partial\right)^{-1}\left(3 Q_{1}^{T} \partial+Q_{1 x}^{T}\right)+\frac{1}{2}\left(Q_{1} \partial^{-1} Q_{2}^{T}\right)^{T} \\
& \quad-\frac{1}{2}\left(Q_{1}^{T} \partial^{-1} Q_{2}+Q_{2}^{T} \partial^{-1} Q_{1}\right) I
\end{aligned}
$$

And then the reduced Hamiltonian operator for the multi-component Novikov equation (2.12) is

$$
\Pi^{\mathrm{red}}\left(Q_{1}, Q_{2}\right)=\left(\begin{array}{l}
\Pi_{11}\left(P_{1}, P_{2}, 0,0\right) \Pi_{12}\left(P_{1}, P_{2}, 0,0\right) \\
\Pi_{21}\left(P_{1}, P_{2}, 0,0\right) \Pi_{22}\left(P_{1}, P_{2}, 0,0\right)
\end{array}\right) .
$$

Moreover, by studying the Hamiltonian operator $\mathscr{K} \mathscr{J}^{-1} \mathscr{K}$ and its Dirac reduction, the second Hamiltonian operator for the multi-component system (2.12) may be obtained as

$$
\Lambda^{\mathrm{red}}\left(Q_{1}, Q_{2}\right)=-\frac{1}{4}\left(\partial^{2}-1\right)\left(\begin{array}{l}
\Pi_{44}\left(P_{1}, P_{2}, 0,0\right) \Pi_{43}\left(P_{1}, P_{2}, 0,0\right) \\
\Pi_{34}\left(P_{1}, P_{2}, 0,0\right) \Pi_{33}\left(P_{1}, P_{2}, 0,0\right)
\end{array}\right)^{-1}\left(\partial^{2}-1\right) .
$$

Especially under the constraint $Q=(q, r)^{T}, R=(r, q)^{T}, U=(u, v)^{T}, V=(v, u)^{T}$, the twocomponent system (2.13) has a Hamiltonian pair

$$
\begin{aligned}
& \Pi^{\mathrm{red}}(q, r)=\frac{1}{2}\left(\begin{array}{c}
3 q \partial+2 q_{x} \\
3 r \partial+2 r_{x}
\end{array}\right)\left(\partial^{3}-4 \partial\right)^{-1}\left(3 q \partial+q_{x} 3 r \partial+r_{x}\right) \\
&+\frac{1}{2}\left(\begin{array}{cc}
q \partial^{-1} q & -q \partial^{-1} r \\
-r \partial^{-1} q & r \partial^{-1} r
\end{array}\right), \\
& \Lambda^{\mathrm{red}}(q, r)=-\frac{1}{4}\left(\partial^{2}-1\right)\left(\begin{array}{cc}
r \partial^{-1} r & q \partial^{-1} r+r \partial^{-1} q \\
q \partial^{-1} r+r \partial^{-1} q & q \partial^{-1} q
\end{array}\right)^{-1}\left(\partial^{2}-1\right) \\
&=-\frac{1}{12}\left(\partial^{2}-1\right)\left(\begin{array}{cc}
-\frac{1}{q} \partial \frac{q}{r} \partial^{-1} \frac{q}{r} \partial \frac{1}{q} & \frac{1}{q}\left(2-\left(\frac{q}{r}\right)_{x} \partial^{-1} \frac{r}{q}\right) \partial \frac{1}{r} \\
\frac{1}{r}\left(2-\left(\frac{r}{q}\right)_{x} \partial^{-1} \frac{q}{r}\right) \partial \frac{1}{q} & -\frac{1}{r} \partial \frac{r}{q} \partial^{-1} \frac{r}{q} \partial \frac{1}{r}
\end{array}\right)\left(\partial^{2}-1\right) .
\end{aligned}
$$

Remark 2.2. The two-component system (2.13) can be written in bi-Hamiltonian form (2.10) with bi-Hamiltonian operators given by (2.14). The associated Hamiltonian functional for $\Pi^{\text {red }}(q, r)$ is $H=\int q v+r u d x$. The omission of $W$ here is caused by the nonlocal property and complication of it. 


\section{Conserved quantities and locality of the Hamiltonians of the Geng-Xue equation (1.4)}

In this section, we give two sequences of conserved quantities for the Geng-Xue equation (1.4), some of them seem to be new. Furthermore, we find all of them are homogeneous and all the Hamiltonians $H_{j}, j \leq-1$ are local.

\subsection{Conserved quantities}

Defining

$$
\varphi=\left(\varphi_{1}, \varphi_{2}, \varphi_{3}\right)^{T}, \quad a=\frac{\varphi_{1}}{\varphi_{2}}, \quad b=\frac{\varphi_{3}}{\varphi_{2}},
$$

based on the Lax pair (1.5), we have

$$
\rho=\left(\ln \varphi_{2}\right)_{x}=\lambda n b
$$

The infinitely many conserved quantities for the Geng-Xue equation (1.4) can be constructed using the standard algorithm. Next we will utilize the procedure in Ref. [30] to obtain the required Hamiltonians.

Substituting these equalities (3.1) to the spectral problem (1.5) yields

$$
a_{x}=\lambda m+b-a \rho, \quad b_{x}=a-b \rho,
$$

after expanding $a, b$ into a series of the spectral parameter $\lambda$ and equating the coefficients of different powers of $\lambda$ in these equations (3.3), one can find a series of conserved densities from coefficients of $\rho$ in power of $\lambda$.

Case 1: Expanding $a, b$ as $a=\sum_{i \geq 1} a_{i} \lambda^{i}, b=\sum_{j \geq 1} b_{j} \lambda^{j}$ and substituting them into the system (3.3), we establish that

$$
\begin{aligned}
& \begin{array}{l}
a_{1}=-u_{x}, \quad b_{1}=-u, \\
\left(1-\partial^{2}\right) b_{3}=\left(n u^{2}\right)_{x}+n u u_{x},
\end{array} \\
& \ldots, \\
& \left(1-\partial^{2}\right) a_{k}=\sum_{i, j \geq 1}^{i+j=k-1}\left[n b_{i} b_{j}+\left(n a_{i} b_{j}\right)_{x}\right], \\
& \qquad\left(1-\partial^{2}\right) b_{k}=\sum_{i, j \geq 1}^{i+j=k-1}\left[n a_{i} b_{j}+\left(n b_{i} b_{j}\right)_{x}\right] .
\end{aligned}
$$

Hence an infinite sequence of conserved quantities are obtained, and the first two are

$$
\begin{aligned}
& H_{1}=\int u n d x \\
& H_{2}=\int\left(u_{x} v-u v_{x}\right) u n d x .
\end{aligned}
$$

Remark 3.1. The above two conserved quantities can be obtained from the Hamiltonian functionals (2.6) $\mathscr{H}_{1}, \mathscr{H}_{2}$ under the constraint $N=1, Q=m, R=n, U=u, V=v$ respectively. 
Case 2: The second expansion is $a=\sum_{i \geq 0} a_{i} \lambda^{\frac{1-2 i}{3}}, b=\sum_{j \geq 0} b_{j} \lambda^{-\frac{2 j+1}{3}}$. Substituting it into the system (3.3) yields:

$$
\begin{aligned}
a_{0}= & m^{\frac{2}{3}} n^{-\frac{1}{3}}, \quad b_{0}=m^{\frac{1}{3}} n^{-\frac{2}{3}}, \\
a_{1}= & -\frac{1}{3}(m n)^{-\frac{2}{3}} m_{x}, \quad b_{1}=\frac{1}{3} m^{-1} n^{-2}\left(m n_{x}-m_{x} n\right), \\
& \ldots, \\
a_{k+1}= & \frac{1}{3}\left[2(m n)^{-\frac{1}{3}}\left(b_{k-1}-a_{k x}\right)+b_{k x}+\sum_{i, j \geq 1}^{i+j=k+1}\left(n b_{i} b_{j}-2 b_{0}^{-1} a_{i} b_{j}\right)\right], \\
b_{k+1}= & \frac{1}{3}(m n)^{-\frac{2}{3}}\left(b_{k-1}-a_{k x}\right)-\frac{1}{3}(m n)^{-\frac{1}{3}}\left[b_{k x}+\sum_{i, j \geq 1}^{i+j=k+1}\left(b_{0}^{-1} a_{i}+n b_{i}\right) b_{j}\right] .
\end{aligned}
$$

In particular, we only consider the conserved quantities associated with $b_{3 k}, k \in \mathrm{Z}$, and obtain the first two conserved quantities as

$$
\begin{aligned}
& H_{-1}=3 \int(m n)^{\frac{1}{3}} d x, \\
& H_{-2}=\frac{1}{27} \int\left[5 m_{x}^{2} n_{x} n^{-\frac{5}{3}} m^{-\frac{8}{3}}-3(m n)^{-\frac{5}{3}} m_{x x} n_{x}-18 n_{x} m^{-\frac{2}{3}} n^{-\frac{5}{3}}\right] d x .
\end{aligned}
$$

The negative powers of $m$ and $n$ that are involved in the above expressions for the conserved quantities raise an important structural property, whose solution established their analytic validity. Namely, we expect that if $m>0$ and $n>0$ hold initially (at time $t=0$ ), then this property is preserved by the Geng-Xue flow. That this might be so is suggested by the granting, in the context of the $\mathrm{CH}$ equation, of such a property by means of a pointwise conservation law that has in that context very strong consequences for the long-time behavior of solutions $[4,20]$. For the Geng-Xue flow we can proceed as follows. Define the smooth diffeomorphisms $\psi(x, t)$ by $\psi(x, 0)=x$ and $\psi_{t}(x, t)=$ $u(\psi(x, t), t) v(\psi(x, t), t)$ for $t>0$ and consider the expressions $\bar{Q}(x, t)=m(\psi(x, t), t) \psi_{x}^{3}(x, t)$ and $\bar{R}(x, t)=n(\psi(x, t), t) \psi_{x}^{3}(x, t)$. Since

$$
\psi_{x t}=\left[u_{x}(\psi, t) v(\psi, t)+u(\psi, t) v_{x}(\psi, t)\right] \psi_{x}, t>0,
$$

and $\psi_{x}(x, 0)=1$ yield

$$
\psi_{x}(x, t)=\exp \left(\int_{0}^{t}\left[u_{x}(\psi, s) v(\psi, s)+u(\psi, s) v_{x}(\psi, s)\right] d s\right)>0, t \geq 0,
$$

using (1.4) we can verify that the relations

$$
\begin{aligned}
& \partial_{t} \bar{Q}(x, t)=3 u(\psi(x, t), t) v_{x}(\psi(x, t), t) \psi_{x}^{3}(x, t) \bar{Q}(x, t), \\
& \partial_{t} \bar{R}(x, t)=3 u_{x}(\psi(x, t), t) v(\psi(x, t), t) \psi_{x}^{3}(x, t) \bar{R}(x, t),
\end{aligned}
$$

hold for any fixed $x$. Therefore

$$
\begin{aligned}
& \bar{Q}(x, t)=\bar{Q}(x, 0) \exp \left(3 \int_{0}^{t}\left[u(\psi(x, s), s) v_{x}(\psi(x, s), s) \psi_{x}^{3}(x, s) d s\right), t \geq 0,\right. \\
& \bar{R}(x, t)=\bar{R}(x, 0) \exp \left(3 \int_{0}^{t}\left[u_{x}(\psi(x, s), s) v(\psi(x, s), s) \psi_{x}^{3}(x, s) d s\right), t \geq 0 .\right.
\end{aligned}
$$


These considerations prove that $m(x, t)>0$ and $n(x, t)>0$ at any $t \geq 0$, provided that $m(x, 0)>0$ and $n(x, 0)>0$.

\subsection{Homogeneous and local properties of the Hamiltonians}

The first nontrivial negative flow in the Geng-Xue hierarchy is the Geng-Xue equation (1.4) [19]. It admits a bi-Hamiltonian structure [29], viz:

$$
\theta_{t}=K \frac{\delta H_{2}}{\delta \theta}=J \frac{\delta H_{1}}{\delta \theta}, \quad \theta=\left(\begin{array}{c}
m \\
n
\end{array}\right)
$$

where $H_{1}, H_{2}$ are given by (3.4), and $K, J$ are respectively the reductions of the Hamiltonian operators $\mathscr{K}, \mathscr{J}(2.4),(2.5)$ under the constraint $N=1, Q=m, R=n, U=u, V=v$, namely

$$
\begin{aligned}
& K=\left(\begin{array}{cc}
0 & \partial^{2}-1 \\
1-\partial^{2} & 0
\end{array}\right), \\
& J=\left(\begin{array}{ll}
J_{11} & J_{12} \\
J_{21} & J_{22}
\end{array}\right)
\end{aligned}
$$

with

$$
\begin{aligned}
J_{11} & =\frac{3}{2} m \partial^{-1} m+\left(m_{x}+\frac{3}{2} m \partial\right)\left(\partial^{3}-4 \partial\right)^{-1}\left(m_{x}+3 m \partial\right), \\
J_{12} & =-\frac{3}{2} m \partial^{-1} n+\left(m_{x}+\frac{3}{2} m \partial\right)\left(\partial^{3}-4 \partial\right)^{-1}\left(n_{x}+3 n \partial\right), \\
J_{21} & =-\frac{3}{2} n \partial^{-1} m+\left(n_{x}+\frac{3}{2} n \partial\right)\left(\partial^{3}-4 \partial\right)^{-1}\left(m_{x}+3 m \partial\right), \\
J_{22} & =\frac{3}{2} n \partial^{-1} n+\left(n_{x}+\frac{3}{2} n \partial\right)\left(\partial^{3}-4 \partial\right)^{-1}\left(n_{x}+3 n \partial\right) .
\end{aligned}
$$

Furthermore, the first nontrivial positive flow in the hierarchy reads

$$
\begin{aligned}
m_{t} & =\left(m^{\frac{1}{3}} n^{-\frac{2}{3}}\right)_{x x}-m^{\frac{1}{3}} n^{-\frac{2}{3}}, \\
n_{t} & =n^{\frac{1}{3}} m^{-\frac{2}{3}}-\left(n^{\frac{1}{3}} m^{-\frac{2}{3}}\right)_{x x},
\end{aligned}
$$

which can also be reformulated as a bi-Hamiltonian form

$$
\theta_{t}=J \frac{\delta H_{-2}}{\delta \theta}=K \frac{\delta H_{-1}}{\delta \theta},
$$

herein $H_{-1}, H_{-2}$ are given by (3.5).

Hence, the Geng-Xue hierarchy may be constructed as

$$
\theta_{t_{j}}=J \frac{\delta H_{j}}{\delta \theta}=K \frac{\delta H_{j+1}}{\delta \theta}, \quad j \in \mathrm{Z}, \quad j \neq 0,-1,
$$

with infinitely many Hamiltonian functionals $H_{j}$ generated by the recursive definition. It is immediately clear that all $H_{j}$ s are homogeneous and $H_{j} \mathrm{~s}, j \geq 3$ are nonlocal. Hence we will discuss the local property of $H_{j}, j \leq-1$. 
Lemma 3.1 ([26,34]). If a differential function $\widehat{M}[\theta]$ satisfies

$$
\int \widehat{M}[\theta] d x=0
$$

for all $\theta$, then there exists a unique differential function $\widehat{N}[\theta]$ up to addition of a constant such that $\widehat{M}[\theta]$ is the total $x$-derivative $\widehat{M}[\theta]=(\widehat{N}[\theta])_{x}$.

Theorem 3.1. Let $X_{j}[\theta]=\frac{\delta H_{j}[\theta]}{\delta \theta}$, then for each $j \leq-1, X_{j}[\theta]$ and $H_{j}[\theta]$ are local.

Proof. We only need to demonstrate the local property of $X_{j}$ s because of $H_{j}[\theta]=\int_{0}^{1} X_{j}[\varepsilon \theta] \cdot \theta d \varepsilon$. As $j=-1, X_{-1}[\theta]$ is local since

$$
X_{-1}[\theta]=\left(m^{-\frac{2}{3}} n^{\frac{1}{3}}, m^{\frac{1}{3}} n^{-\frac{2}{3}}\right)^{T} .
$$

Now suppose that $X_{j}[\theta]$ is local for $j=-k$. When $j=-(k+1)$, we have

$$
X_{-(k+1)}[\theta]=J^{-1} K X_{-k}[\theta]=\left(J^{-1} K\right)^{k} X_{-1}[\theta] .
$$

To find an explicit expression for $X_{-k}[\theta]$, a direct choice is to calculate the inverse operator of $J$. However, it seems to be difficult. Hence, we find an indirect way. Suppose $h=(m n)^{\frac{1}{3}}$ and define

$$
\begin{aligned}
& X_{-k}[\theta]=\left(A_{k}, B_{k}\right)^{T}, \\
& E_{k}=\left(\partial^{3}-4 \partial\right)^{-1}\left(3 m \partial+m_{x}, 3 n \partial+n_{x}\right) X_{-k}[\theta],
\end{aligned}
$$

it follows from the recursive relation (3.11) that

$$
\begin{aligned}
& \frac{3}{2} m \partial^{-1}\left(m A_{k+1}-n B_{k+1}\right)+\left(\frac{3}{2} m \partial+m_{x}\right) E_{k+1}=\left(\partial^{2}-1\right) B_{k}, \\
& -\frac{3}{2} n \partial^{-1}\left(m A_{k+1}-n B_{k+1}\right)+\left(\frac{3}{2} n \partial+n_{x}\right) E_{k+1}=\left(1-\partial^{2}\right) A_{k} .
\end{aligned}
$$

Eliminating $E_{k+1}$ from the above system and combating the definition of $E_{k+1}$ yield

$$
\left(\begin{array}{cc}
3 m \partial+m_{x} & 3 n \partial+n_{x} \\
m & -n
\end{array}\right)\left(\begin{array}{l}
A_{k+1} \\
B_{k+1}
\end{array}\right)=\frac{1}{3} \Theta\left(\begin{array}{l}
A_{k} \\
B_{k}
\end{array}\right)
$$

where

$$
\Theta=\left(\begin{array}{cc}
0 & 0 \\
0 & \partial \frac{2}{m}\left(\partial^{2}-1\right)
\end{array}\right)+\left(\begin{array}{c}
4 \partial-\partial^{3} \\
\partial^{2}+\partial \frac{2 m_{x}}{3 m}
\end{array}\right) \frac{1}{h} \partial^{-1}\left(\begin{array}{c}
-m^{\frac{1}{3}} n^{-\frac{2}{3}} \\
m^{-\frac{2}{3}} n^{\frac{1}{3}}
\end{array}\right)\left(1-\partial^{2}\right) .
$$

Then it is not difficult to solve $A_{k+1}$ and $B_{k+1}$ from the above system, and we find that $A_{k+1}$ and $B_{k+1}$ are local if there exist two differential functions $M_{k}, N_{k}$ such that

$$
\begin{aligned}
\Gamma_{1} & =\frac{1}{h^{2}}\left[m\left(1-\partial^{2}\right) A_{k}+n\left(\partial^{2}-1\right) B_{k}\right]=M_{k x}, \\
\Gamma_{2} & =\frac{1}{h}\left(\left[\partial^{3}-4 \partial+3\left(\partial-\frac{2 m_{x}}{3 m}\right) \partial\left(\partial+\frac{2 m_{x}}{3 m}\right)\right] \frac{M_{k}}{h}-\left(\partial-\frac{2 m_{x}}{3 m}\right) \partial \frac{6}{m}\left(\partial^{2}-1\right) B_{k}\right) \\
& =N_{k x} .
\end{aligned}
$$


Using the Lemma 3.1, the equality (3.13) follows immediately from

$$
\begin{aligned}
\int \Gamma_{1} d x & =\int \frac{1}{h^{2}}\left[m\left(1-\partial^{2}\right) A_{k}+n\left(\partial^{2}-1\right) B_{k}\right] d x \\
& =\int X_{-1}^{T} K X_{-k} d x \\
& =\int X_{-1}^{T} K\left(J^{-1} K\right)^{k-1} X_{-1} d x \\
& =-\int X_{-1}^{T}\left(K J^{-1}\right)^{k-1} K X_{-1} d x \\
& =-\int X_{-1}^{T} K\left(J^{-1} K\right)^{k-1} X_{-1} d x \\
& =-\int X_{-1}^{T} K X_{-k} d x,
\end{aligned}
$$

where we have used the skew-symmetric property of $K, J$. So $\int \Gamma_{1} d x=0$. Similarly, the equality (3.14) may be demonstrated from

$$
\begin{aligned}
\int \Gamma_{2} d x= & \int \frac{1}{h}\left[\partial^{3}-4 \partial+\left(3 \partial-2 \frac{m_{x}}{m}\right)\left(\partial^{2}+\partial \frac{2 m_{x}}{3 m}\right)\right] \frac{M_{k}}{h} \\
& -\frac{1}{h}\left(3 \partial-2 \frac{m_{x}}{m}\right) \partial \frac{2}{m}\left(\partial^{2}-1\right) B_{k} d x \\
= & \int f\left(1-\partial^{2}\right) A_{k}+g\left(\partial^{2}-1\right) B_{k} d x \\
= & \int(g, f) K\left(A_{k}, B_{k}\right)^{T} d x \\
= & -\int\left(A_{k}, B_{k}\right) K(g, f)^{T} d x \\
= & -\int J^{-1} K\left(A_{k}, B_{k}\right)^{T} J(g, f)^{T} d x=0,
\end{aligned}
$$

where

$$
\begin{aligned}
& f=\frac{1}{h^{4}}\left(\frac{2}{3} m_{x x}-\frac{4}{3} \frac{m n_{x x}}{n}-\frac{10}{9} \frac{m_{x}^{2}}{m}+\frac{14}{9} \frac{m n_{x}^{2}}{n^{2}}+\frac{4}{9} \frac{m_{x} n_{x}}{n}-2 m\right), \\
& g=\frac{1}{h^{4}}\left(\frac{2}{3} n_{x x}-\frac{4}{3} \frac{n m_{x x}}{m}-\frac{10}{9} \frac{n_{x}^{2}}{n}+\frac{14}{9} \frac{n m_{x}^{2}}{m^{2}}+\frac{4}{9} \frac{m_{x} n_{x}}{m}-2 n\right) .
\end{aligned}
$$

In the above we have eliminated the derivatives of $M_{k}$ in the integration by the equality (3.13) and used the skew-symmetric property of $K, J$, as well as the fact that $(g, f)^{T}$ is a kernel of $J$. So all the Hamiltonians $H_{j}, j \leq-1$ for the Geng-Xue equation (1.4) are local.

\section{Acknowledgments}

This work is partially supported by the National Natural Science Foundation of China (Grant Nos. 11805071 and 11747010) and the Initial Founding of Scientific Research for the introduction of talents of Huaqiao University (Project No. 16BS513).

\section{References}

[1] R. Camassa and D.D. Holm, An integrable shallow water equation with peaked solitons, Phys. Rev. Lett. 71 (1993) 1661-1664. 
[2] R. Camassa, D.D. Holm and J.M. Hyman, A new integrable shallow water equation, Adv. Appl. Mech. 31 (1994) 1-31.

[3] A. Constantin, Existence of permanent and breaking waves for a shallow water equation: a geometric approach, Ann. Inst. Fourier 50 (2000) 321-362.

[4] A. Constantin, Finite propagation speed for the Camassa-Holm equation, J. Math. Phys. 46 (2005) 023506.

[5] A. Constantin, On the scattering problem for the Camassa-Holm equation, Proc. Roy. Soc. London A 457 (2001) 953-970.

[6] A. Constantin, Particle trajectories in extreme Stokes waves, IMA J. Appl. Math. 77 (2012) 293-307.

[7] A. Constantin, The trajectories of particles in Stokes waves, Invent. Math. 166 (2006) 523-535.

[8] A. Constantin and J. Escher, On the blow-up rate and the blow-up set of breaking waves for a shallow water equation, Math. Z. 233 (2000) 75-91.

[9] A. Constantin and J. Escher, Particle trajectories in solitary water waves, Bull. Amer. Math. Soc. 44 (2007) 423-431.

[10] A. Constantin and J. Escher, Wave breaking for nonlinear nonlocal shallow water equations, Acta Math. 181 (1998) 229-243.

[11] A. Constantin, V.S. Gerdjikov and R.I. Ivanov, Inverse scattering transform for the Camassa-Holm equation, Inverse Problems 22 (2006) 2197-2207.

[12] A. Constantin, R.I. Ivanov and J. Lenells, Inverse scattering transform for the Degasperis-Procesi equation, Nonlinearity 23 (2010) 2559-2575.

[13] A. Constantin and D. Lannes, The hydrodynamical relevance of the Camassa-Holm and DegasperisProcesi equations, Arch. Ration. Mech. Anal. 192 (2009) 165-186.

[14] A. Constantin and H.P. McKean, A shallow water equation on the circle, Comm. Pure Appl. Math. 52 (1999) 949-982.

[15] A. Constantin and W. Strauss, Stability of peakons, Comm. Pure Appl. Math. 53 (2000) 603-610.

[16] A. Degasperis, D.D. Holm and A.N.W. Hone, A new integrable equation with peakon solitons, Theor. Math. Phys. 133 (2002) 1463-1474.

[17] A. Degasperis and M. Procesi, Asymptotic integrability Symmetry and Perturbation Theory ed. A. Degasperis and G. Gaeta (Singapore, World Scientific, 1999) pp. 23-37.

[18] B. Fuchssteiner, Some tricks from the symmetry-toolbox for nonlinear equations: generalizations of the Camassa-Holm equation, Phys. D 95 (1996) 229-243.

[19] X.G. Geng and B. Xue, An extension of integrable peakon equations with cubic nonlinearity, Nonilinearity 22 (2009) 1847-1856.

[20] D. Henry, Compactly supported solutions of the Camassa-Holm equation, J. Nonlinear Math. Phys. 12 (2005) 342-347.

[21] A.N.W. Hone and J.P. Wang, Integrable peakon equations with cubic nonlinearity, J. Phys. A: Math. Theor. 41 (2008) 372002.

[22] A.N.W. Hone and J.P. Wang, Prolongation algebras and Hamiltonian operators for peakon equations, Inverse Problems 19 (2003) 129-145.

[23] D. Ionescu-Kruse, Variational derivation of the Camassa-Holm shallow water equation, J. Nonlinear Math. Phys. 14 (2007) 311-320.

[24] R.S. Johnson, Camassa-Holm, Korteweg-de Vries and related models for water waves. J. Fluid Mech. 455 (2002) 63-82.

[25] J. Lenells, A variational approach to the stability of periodic peakons, J. Nonlinear Math. Phys. 11 (2004) 151-163.

[26] J. Lenells, Conservation laws of the Camassa-Holm equation, J. Phys. A: Math. Gen. 38 (2005) 869880.

[27] H.M. Li and W. Chai, A new Liouville transformation for the Geng-Xue system, Commun. Nonlinear Sci. Numer. Simul. 49 (2017) 93-101.

[28] H.M. Li, Y.Q. Li and Y. Chen, Bi-Hamiltonian structure of multi-component Novikov equation, $J$. Nonlinear Math. Phys. 21 (2014) 509-520. 
[29] N.H. Li and Q.P. Liu, On bi-Hamiltonian structure of two-component Novikov equation, Phys. Lett. A 377 (2013) 257-261.

[30] N.H. Li and X.X. Niu, A reciprocal transformation for the Geng-Xue equation, J. Math. Phys. 55 (2014) 053505.

[31] Y. Liu and Z. Lin, Stability of peakons for the Degasperis-Procesi equation, Comm. Pure Appl. Math. 62 (2009) 125-146.

[32] V.S. Novikov, Generalizations of the Camassa-Holm equation, J. Phys. A: Math. Theor. 42 (2009) 342002.

[33] W. Oevel and O. Ragnisco, R-matrices and higher poisson brackets for integrable systems, Phys. A 161 (1989) 181-220.

[34] P.J. Olver, Applications of Lie Groups to Differential Equations (Springer, Berlin, 1993).

[35] J.F. Toland, Stokes waves, Topol. Methods Nonlinear Anal. 7 (1996) 1-48. 